\title{
Langmuir
}

pubs.acs.org/Langmuir

(C) 2010 American Chemical Society

\section{Flat-On Lamellae in Spin-Coated, Stable Films of Poly(propylene azelate)}

\author{
D. R. Rueda,* J. J. Hernández, M. C. García-Gutiérrez, and T. A. Ezquerra \\ Instituto de Estructura de la Materia, CSIC. Serrano 121, 28006 Madrid, Spain \\ M. Soccio, N. Lotti, and A. Munari \\ Dipartimento di Ingegneria Civile, Ambientale e dei Materiali, Universitá di Bologna, Via Terracini 28, \\ 40131 Bologna, Italy \\ J. Perlich
}

DESY-HASYLAB, Notkestrasse 85, 22607 Hamburg, Germany

R. Serna

Instituto de Optica, Serrano 121, 28006 Madrid, Spain

Received July 29, 2010. Revised Manuscript Received September 20, 2010

\begin{abstract}
Thin films (5-500 nm thick) of the linear aliphatic polyester $(3,7)$ poly(propylene azelate) (PPAz) were prepared by spin-coating of $\mathrm{CHCl}_{3}$ polymer solutions with different polymer concentrations. The morphology and structure of the spin-coated thin films were investigated by atomic force microscopy (AFM) and by grazing incidence wide-angle X-ray scattering (GIWAXS) techniques. AFM revealed the continuous nature of the flat, spherulitic films which are stable against dewetting even for polymer coatings as thin as $15 \mathrm{~nm}$. GIWAXS patterns revealed a high crystal orientation of the films. A sharp reflection on the meridian whose spacing is related to the polymer chain unit length (c-axis) supports the presence of flat-on lamellae morphology in the whole range of film thicknesses investigated. The flat-on lamellae morphology is also supported by AFM images. A triclinic unit cell with the $c^{*}$-axis perpendicular to the substrate is proposed for PPAz. The repulsion of the long aliphatic spacer by the Si-substrate is invoked as the main reason for the flat-on morphology observed.
\end{abstract}

\section{Introduction}

Thin polymer films introduce two-dimensional spatial confinement which may affect the molecular order, ${ }^{1}$ the crystallization behavior, ${ }^{2,3}$ the morphology, ${ }^{4}$ or even the crystalline structure ${ }^{5}$ of the polymer. One important aspect in thin films of semicrystalline polymers is related to the orientation of the crystalline lamellae. It has been generally observed that thin films of several hundreds of nanometers exhibit predominantly edge-on lamellae while ultrathin films exhibit flat-on lamellae. Nevertheless, there are several factors, besides thickness, ${ }^{6}$ such as the interaction with the substrate $^{3}$ or the crystallization temperature ${ }^{4}$ that may control the relative abundance of different crystalline lamellae orientations.

Aliphatic polyesters are very attractive materials because they may combine properties of biocompatibily and biodegradablility

(1) Rueda, D. R.; Nogales, A.; Hernandez, J. J.; Garcia-Gutierrez, M. C.; Ezquerra, T. A.; Roth, S. V.; Zolotukhin, M. G.; Serna, R. Stacking of main chaincrown ether polymers in thin films. Langmuir 2007, 23(25), 12677-12681.

(2) Capitan, M. J.; Rueda, D. R.; Ezquerra, T. A. Inhibition of the crystallization in nanofilms of poly(3-hydroxybutyrate). Macromolecules 2004, 37(15), $5653-5659$.

(3) Ma, Y.; Hu, W. B.; Reiter, G. Lamellar crystal orientations biased by crystallization kinetics in polymer thin films. Macromolecules 2006, 39(15), 51595164 .

(4) Wang, Y.; Chan, C. M.; Ng, K. M.; Li, L. What controls the lamellar orientation at the surface of polymer films during crystallization? Macromolecules 2008, 41(7), 2548-2553.

(5) Park, Y. J.; Kang, S. J.; Lotz, B.; Brinkmann, M.; Thierry, A.; Kim, K. J.; Park, C. Ordered ferroelectric PVDF-TrFE thin films by high throughput epitaxy for nonvolatile polymer memory. Macromolecules 2008, 41(22), 8648-8654.

(6) Liang, Y. R.; Zheng, M. Z.; Park, K. H.; Lee, H. S. Thickness-dependent crystal orientation in poly(trimethylene 2,6-naphthalate) films studied with GIWAXD and RA-FTIR methods. Polymer 2008, 49(7), 1961-1967. with other physical and chemical properties similar to those of commodity polymers. ${ }^{7-9}$ Those aliphatic polyesters based on propylene glycol became of growing interest because this component is obtained from renewable sources at relatively low cost. ${ }^{9,10}$

Previously, ${ }^{11}$ we have reported on the structure and morphology observed in spin-coated thin films of a series of linear aliphatic polyesters [-O $\left.-\left(\mathrm{CH}_{2}\right)_{3}-\mathrm{O}-\mathrm{CO}-\left(\mathrm{CH}_{2}\right)_{Y}-\mathrm{CO}-\right]$, based on propylene glycol and a dicarboxylic acid of different length (aliphatic spacer, $Y=2,3$, and 4). There, we showed that spin-coated films thinner than $\sim 100 \mathrm{~nm}$ tend to be unstable and spontaneously dewet at room temperature. This fact prevents the use of these aliphatic polyesters of short aliphatic spacer $(Y \leq 4)$ as stable ultrathin films for practical use in the biomedical field. ${ }^{12-14}$

(7) Martinez-Palau, M.; Franco, L.; Puiggali, J. Isothermal crystallization of poly(glycolic acici-alt-6-hydroxyhexanoic acid) studied by DSC and real time synchrotron SAXS/WAXD. Polymer 2007, 48(20), 6018-6028.

(8) Gesti, S.; Zanetti, M.; Lazzari, M.; Franco, L.; Puiggali, J. Degradable polyoctamethylene suberate/clay nanocomposites. Crystallization studies by DSC and simultaneous SAXS/WAXD synchrotron radiation. Eur. Polym. J. 2009, 45 (2), 398-409.

(9) Soccio, M.; Lotti, N.; Finelli, L.; Gazzano, M.; Munari, A. Aliphatic poly(propylene dicarboxylate)s: Effect of chain length on thermal properties and crystallization kinetics. Polymer 2007, 48(11), 3125-3136.

(10) Soccio, M.; Lotti, N.; Finelli, L.; Gazzano, M.; Munari, A. Neopenthyl glycol containing poly(propylene azelate)s: Synthesis and thermal properties. Eur. Polym. J. 2007, 43(8), 3301-3313.

(11) Hernandez, J. J.; Rueda, D. R.; Garcia-Gutierrez, M. C.; Nogales, A.; Ezquerra, T. A.; Soccio, M.; Lotti, N.; Munari, A. Structure and morphology of thin films of linear aliphatic polyesters prepared by spin-coating. Langmuir $\mathbf{2 0 1 0}$, 26(13), 10731-10737.

(12) Segalman, R. A.; Green, P. F. Dynamics of rims and the onset of spinodal dewetting at liquid/liquid interfaces. Macromolecules 1999, 32(3), 801-807. 
Table 1. Polymer Solution Concentration, Film Thickness as Measured by Ellipsometry, and fwhm Values Measured from the Azimuth Profiles of the 001 Reflection for the PPAz Spin-Coating Samples Investigated

sample concentration (g/L) film thickness (nm) fwhm_(001) (deg)

$\begin{array}{lrc}(1: 0) & 40.0 & 534 \pm 27 \\ (1: 1) & 20.0 & 136 \pm 7 \\ (1: 2) & 13.3 & 103 \pm 5 \\ (1: 4) & 8.0 & 58 \pm 3 \\ (1: 8) & 4.4 & 27.4 \pm 1.5 \\ (1: 16) & 2.3 & 14.8 \pm 0.7 \\ (1: 32) & 1.2 & 18.7 \pm 0.7 \\ (1: 64) & 0.6 & 4.6 \pm 0.2\end{array}$

8.2

1367

$03 \pm 5$

$58+3$

$4.8 \pm 0.7$

$4.6 \pm 0.2$

This Article reports on the structure and morphology observed for the spin-coated samples of the linear aliphatic polyester $(3,7)$ poly(propylene azelate). A combined study by means of atomic force microscopy (AFM) and grazing incidence wide angle X-rays scattering (GIWAXS) has revealed a clear, flat-on lamellae morphology. The observed stability of films as thin as $15 \mathrm{~nm}$ against dewetting is remarkable. According to the peak sharpness of the 001 reflection in its azimuth direction, we can affirm that samples of thickness $\leq 60 \mathrm{~nm}$ exhibit a similar and full lamellar orientation.

\section{Experimental Section}

2.1. Materials and Sample Preparation. Poly(propylene azelate) (PPAz) is a linear aliphatic polyester with chain repeating unit: $\left[-\mathrm{O}-\left(\mathrm{CH}_{2}\right)_{3}-\mathrm{O}-\mathrm{CO}-\left(\mathrm{CH}_{2}\right)_{7}-\mathrm{CO}-\right]$. PPAz was synthesized by using the two-stage polycondensation procedure as previously reported. ${ }^{9}$ The PPAz investigated here has a molecular weight of $M_{\mathrm{n}}=36000 \mathrm{~g} / \mathrm{mol}$ and a polydispersity degree of 2.5 . It is characterized by a low melting temperature $\left(T_{\mathrm{m}}=49^{\circ} \mathrm{C}\right)$ and a glass transition $\left(T_{\mathrm{g}}=-56{ }^{\circ} \mathrm{C}\right)$ far below room temperature. ${ }^{9}$

Thin polymer films were prepared by spin-coating a polymer solution in chloroform $\left(\mathrm{CHCl}_{3}\right)$ on silicon wafers (about $19 \times$ $25 \mathrm{~mm}^{2}$ ) in the following way. First, a polymer mother solution $(40 \mathrm{~g} / \mathrm{L})$, referred hereafter as (1:0), was prepared; other less concentrated solutions were obtained by selective dilution of the mother solution. The following polymer solutions were prepared: $20 \mathrm{~g} / \mathrm{L}(1: 1), 13.3 \mathrm{~g} / \mathrm{L}(1: 2), 8 \mathrm{~g} / \mathrm{L}(1: 4), 4.4 \mathrm{~g} / \mathrm{L}(1: 8), 2.3 \mathrm{~g} / \mathrm{L}(1: 16)$, $1.2 \mathrm{~g} / \mathrm{L}(1: 32)$, and $0.6 \mathrm{~g} / \mathrm{L}$ (1:64). Subsequently, by means of a syringe, $0.1 \mathrm{~mL}$ of a given solution was instantly dropped on the center of a silicon (100) substrate rotating at a speed of $40 \mathrm{~Hz}$ $(2400 \mathrm{rpm})$ for $30 \mathrm{~s}$. Silicon wafers were obtained from Wafer World, Inc. They had both surfaces polished and were used as supplied, with a native silicon oxide surface of $\approx 2 \mathrm{~nm}$ in thickness as determined by ellipsometry, ${ }^{1}$ after cleaning with soap solution (Procter\&Gamble) and bidistilled water. The thickness of the spin-coated films, as measured by ellipsometry assuming a refractive index of 1.4916, increases with the polymer concentration used, as expected. ${ }^{11}$ The variation of film thickness due to nonhomogeneity of samples was estimated to be $5 \%$ (Table 1 ).

2.2. Atomic Force Microscopy. A Nanoscope IIIA Multimode microscope from Veeco operating in the taping mode was used to investigate the surface morphology of the spin-coated films at room temperature. AFM images with different magnifications were obtained and conveniently analyzed. Thus, height profiles revealed sample information about the surface roughness and some other lamellae features.

2.3. Grazing Incidence X-ray Scattering. Grazing incidence small-angle X-ray scattering (GISAXS) and GIWAXS were accomplished by using synchrotron radiation at the synchro-

(13) Seemann, R.; Herminghaus, S.; Jacobs, K. Dewetting patterns and molecular forces: A reconciliation. Phys. Rev. Lett. 2001, 86(24), 5534-5537.

(14) Seemann, R.; Herminghaus, S.; Neto, C.; Schlagowski, S.; Podzimek, D. Konrad, R. Mantz, H. Jacobs, K. Dynamics and structure formation in thin polymer melt films. J. Phys.: Condens. Matter 2005, 17(9), S267-S290.
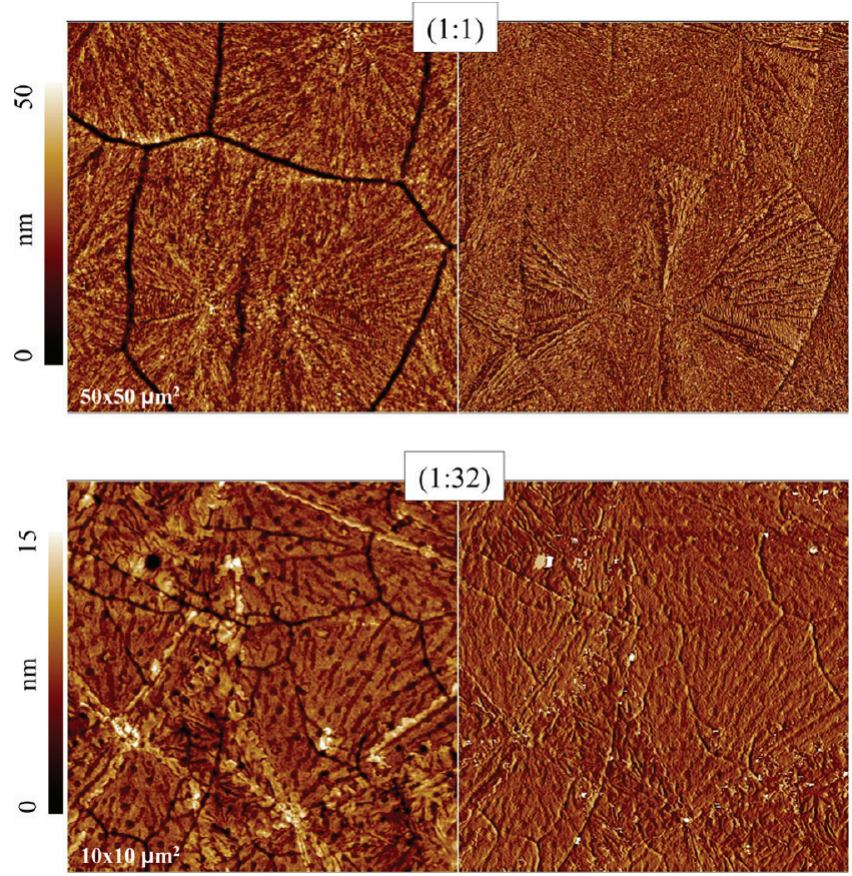

Figure 1. Representative AFM images of the height (left) and phase (right) for two spin-coating samples of PPAz. The polymer solution used is indicated.

tron beamline BW4 of HASYLAB (Hamburg, Germany) ${ }^{15-17}$ using a wavelength $\lambda=0.1381 \mathrm{~nm}$. A moderately focused microbeam with a beam size of $20 \times 40 \mu \mathrm{m}^{2}$ (vertical $\times$ horizontal) was applied. A Mar CCD 165 detector $(2048 \times 2048$ pixels $)$ with a pixel size of $79.1 \times 79.1 \mu \mathrm{m}^{2}$ was used. For GISAXS and GIWAXS experiments, a sample-to-detector distance of 2.043 and $0.1856 \mathrm{~m}$, respectively, was used. Calibration was done by using a standard of silver behenate $\left(d_{001}=5.8380 \mathrm{~nm}\right) .{ }^{18}$ The incidence angle $\alpha_{\mathrm{i}}$ $\left(0.4^{\circ}\right)$ was selected to be twice the critical angle for the silicon substrate which is larger than that for polymer materials. Both GISAXS and GIWAXS patterns were analyzed by using the Fit2D software. ${ }^{19}$ It is worth mentioning the lack of outof-meridian signals in the GISAXS patterns for all the samples investigated. Consequently, we will focus on the GIWAXS results only.

\section{Results and Discussion}

3.1. Morphology of the Spin-Coated PPAz Thin Films as Revealed by AFM. Figure 1 shows representative AFM (height and phase) images for samples prepared from (1:1) and (1:32) polymer solutions. The presence of big, flat spherulites is a common feature for the continuous coated films observed for all the samples investigated, with the only exception of the thinnest sample (1:64). Indeed, for the last sample, a lack of full coverage of the substrate is observed (Figure 2b). It is worth noticing that the presence of dark spots in the topography picture

(15) Bauer, E.; Maurer, E.; Mehaddene, T.; Roth, S. V.; Muller-Buschbaum, P. Flow in confined geometry introduced by dewetting of ultrathin polystyrene films. Macromolecules 2006, 39(15), 5087-5094.

(16) Busch, P.; Posselt, D.; Smilgies, D. M.; Rheinlander, B.; Kremer, F.; Papadakis, C. M. Lamellar diblock copolymer thin films investigated by tapping mode atomic force microscopy: Molar-mass dependence of surface ordering. Macromolecules 2003, 36(23), 8717-8727.

(17) Müller-Buschbaum, P. Lecture Notes in Physics: Applications of Synchrtron Light to Scattering and Diffraction in Materials and Life Sciences; Springer: Berlin, 2009; Vol. 776, p 61

(18) Huang, T. C.; Toraya, H.; Blanton, T. N.; Wu, Y. X-ray-powder diffraction analysis of silver behenate, a possible low-angle diffraction standard. J. Appl. Crystallogr. 1993, 26, 180-184.

(19) http://www.esrf.eu/computing/scientific/FIT2D/. 

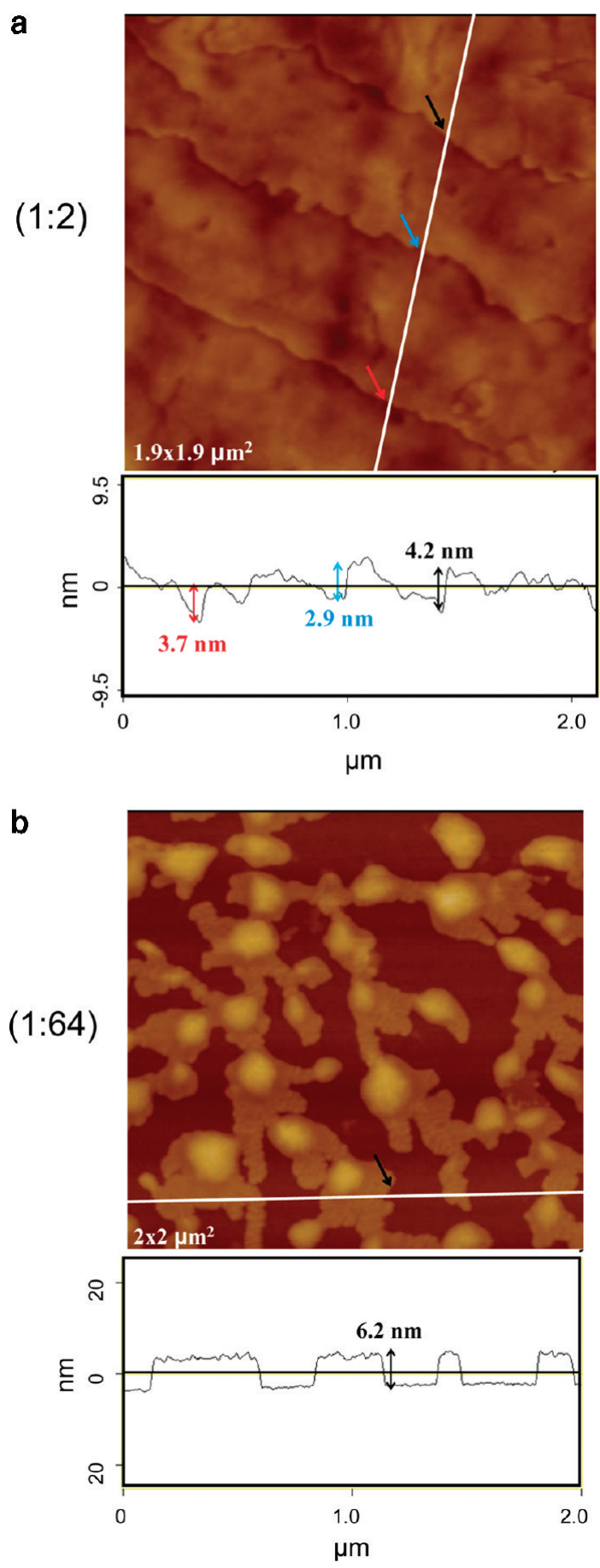

Figure 2. AFM height images (top) and selected height profiles (bottom) for the samples (1:2) and (1:64) to illustrate the flat-on lamellae morphology of PPAz samples.

of sample (1:32) (Figure 1 bottom, left) could be attributed to pores in the film which are precursors for film dewetting. According to its height profile (Figure 2b, bottom), the polymer coating appears to be in flat islands of about $6 \mathrm{~nm}$ in thickness. In Figure 2a, the morphology of a thicker sample (1:2) as well as a height profile are shown. It reveals the average planarity of the film which consists of lamellae stacked on each other. Along the height profile, we observe the step associated to the lamellae edges indicated by arrows on the AFM image. This flat-on-lamellae morphology observed for all PPAz samples is in contrast with the preferential edge-on lamellae reported for the homologous linear aliphatic polyesters of shorter chain lengths $\left(Y=2,3\right.$, and $4 \mathrm{CH}_{2}$

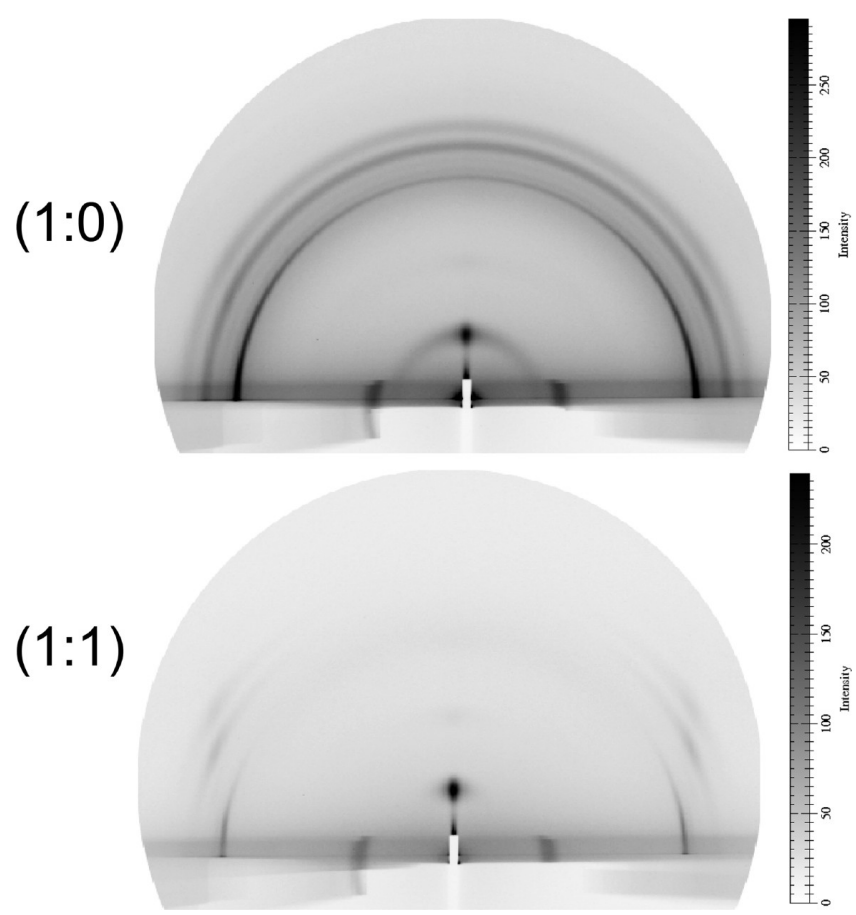

Figure 3. GIWAXS patterns for the two thickest spin-coated samples of PPAz investigated: (1:0) and (1:1).

units of dicarboxylic acid). ${ }^{11}$ In addition, because of dewetting, drop morphology was observed for the thinner samples ${ }^{11}$ in contrast to the flat islands observed for PPAz (1:64) (Figure 2b). If only long-range van der Waals forces are considered, the stability of the system substrate/polymer/air (1/3/2) may be decided upon the sign of the Hamaker constant, $A_{132}$, of the system. $A_{132}$ can be calculated from the Hamaker constant derived for the substrate, $A_{11}$, and the coated polymer, $A_{33}$, according to the expression: $A_{132}=\sqrt{ } A_{33}\left(\sqrt{ } A_{33}-\sqrt{ } A_{11}\right) \cdot{ }^{20}$ In our case, the Si wafer is covered by a thin layer of $\mathrm{SiO}_{2}$ for which $A_{11} \approx 6.5 \times 10^{-20} \mathrm{~J}$. Since the $A_{33}$ value derived for linear aliphatic polyesters is around $17 \times 10^{-20}$ $\mathrm{J}$, it comes out that $A_{132}>0$. Consequently, the spin-coated thin films of PPAz and related aliphatic polyesters on the Si wafer used here would be unstable and could exhibit dewetting. Therefore, the continuous films observed for PPAz should be attributed to other reasons as will be mentioned further below.

3.2. Structure and Chain Orientation as Revealed by GIWAXS. In Figure 3, we have presented the GIWAXS patterns for the two thicker samples (1:0) and (1:1). They reveal the semicrystalline nature of films and a crystal orientation which is evident also for the thickest sample. For this sample (1:0), it is remarkable the presence on the meridian of both a spot reflection and an arc reflection with the same lattice spacing. $(d=1.356 \mathrm{~nm}$ to be commented afterward). For clarity of presentation, Figure 4 shows a GIWAXS pattern revealing the high orientation achieved for a coating about $60 \mathrm{~nm}$ thick. Similar patterns were observed for the rest of the thinner PPAz samples. We will use this pattern for indexing reflections and to clarify the chain orientation in these spin-coated polymer films. A GIWAXS pattern is equivalent to half of a WAXS pattern whose equator and meridian lines are parallel and perpendicular to the surface film plane. The horizontal red line of Figure 4 corresponds to the equator. The GIWAXS patterns obtained, however, exhibit both a horizontal

(20) Bollinne, C.; Cuenot, S.; Nysten, B.; Jonas, A. M. Spinodal-like dewetting of thermodynamically-stable thin polymer films. Eur. Phys. J. E 2003, 12(3), 389-395. 


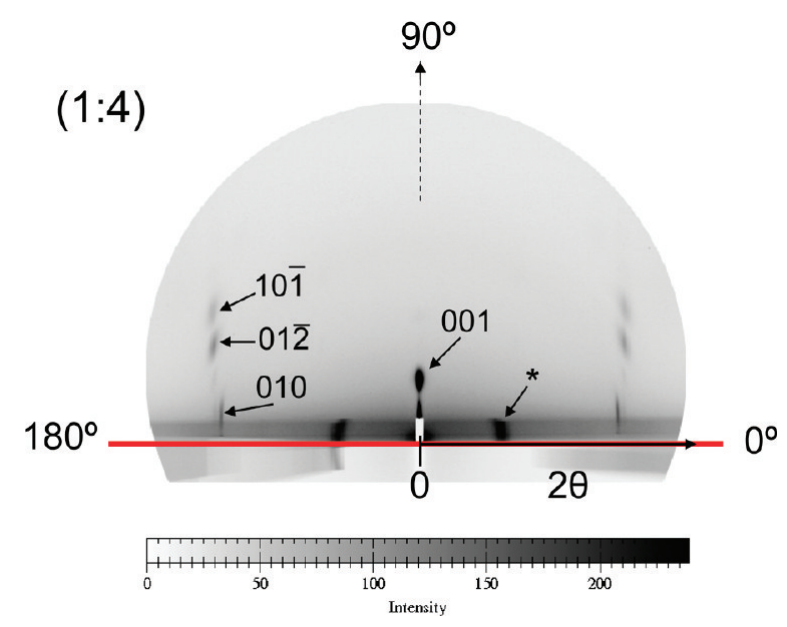

Figure 4. Representative GIWAXS pattern for a highly oriented sample (1:4). The equator (red line), azimuth angle values, and $h \mathrm{kl}$ indexes of main reflections are indicated. The reflection marked with the asterisk $(*)$ arises from Kapton (see text).

band near the equator and a background which will interfere with the analysis of patterns. First of all, we must discard the reflection marked in the figure with the asterisk $(*)$ to be related to the Kapton foil used as window in the vacuum path. The longest lattice spacing observed $(d=1.356 \mathrm{~nm})$ corresponds to the reflection on the meridian. Then, this reflection should be associated to the chain periodicity ( $c$-axis). The highly oriented pattern of Figure 4 reveals the absence of reflections on the equator. Particularly, the reflection closest to the equator has a spacing value of $0.442 \mathrm{~nm}$. This value is similar to the spacing of the strong equatorial reflection indexed as 020 of the monoclinic crystal unit cell (with $b$ as the principal axis) proposed for the homologous aliphatic polyesters. ${ }^{11}$ Therefore, we have considered this reflection to define the $b$-axis of the crystal unit cell. Since both reflections appear not orthogonal to each other and are used to define the $b^{*}$ and $c^{*}$ reciprocal axes, we are proposing for PPAz a triclinic unit cell with parameters $a=$ $0.4778 \mathrm{~nm}, b=0.4480 \mathrm{~nm}, c=1.5110 \mathrm{~nm}, \alpha=95^{\circ}, \beta=65^{\circ}$, and $\gamma=85^{\circ}$. The expression for calculating the lattice spacing for a triclinic crystal unit cell was used. ${ }^{21}$ Accordingly, the corresponding $h k l$ indexes of more intense reflections are indicated in Figure 4.

In order to get more information from the GIWAXS patterns, the scattering intensity curves obtained by their azimuth integration are considered. For clarity reasons, we have also presented separately the scattering curves corresponding to a full $\left(0^{\circ}-180^{\circ}\right)$ azimuth integration (Figure 5, top) and those ones from a partial $\left(70^{\circ}-110^{\circ}\right)$ azimuth integration around the meridian (Figure 5, bottom). On these two plots, we have also indicated the $h \mathrm{kl}$ indexes of reflections already shown in the pattern (Figure 4) along with some others $(002,100$, and 103$)$ of weaker reflections. At first sight, sample (1:0) shows much larger scattering intensity than the rest of samples because of its relatively higher thickness $(530 \mathrm{~nm})$. For this sample, the presence of higher scattering intensity even on the meridian of the near equatorial reflection 010 (see bottom plot of Figure 5) is remarkable. Therefore, it is plausible to assume that the outer layers of the thickest film have a significant lower crystal orientation degree than those lying deeper in thickness. Because of the much larger scattering observed for sample (1:0) in relation to those for thinner samples, the structural features from thin samples are visually reduced. Nevertheless, Bragg reflections (Figure 5, top) are present for all curves as well as the amorphous broad maximum around $q=14 \mathrm{~nm}^{-1}$

(21) Cullity, B. D. Elements of X-ray diffraction; Addison-Wesley: Reading, MA, 1956.
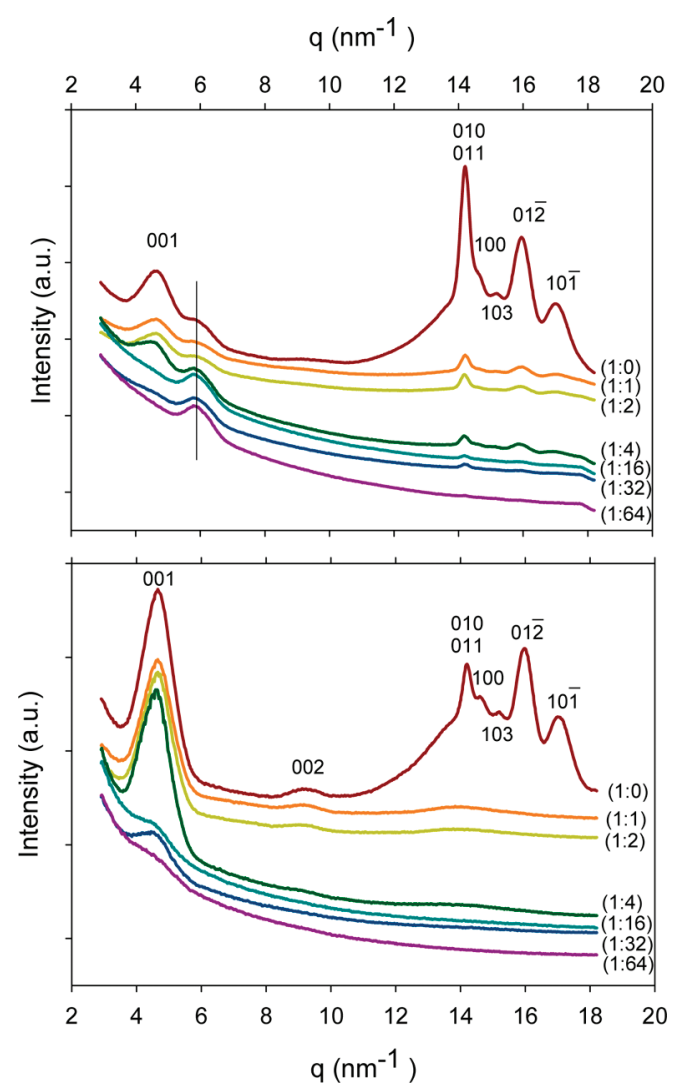

Figure 5. Intensity curves obtained by azimuth integration of the GIWAXS patterns. (Top) Full azimuth range $\left(0^{\circ}-180^{\circ}\right)$. (Bottom) Azimuth interval around the meridian $\left(70^{\circ}-110^{\circ}\right) . h k l$ indexes are indicated. The vertical line points out the reflection related to Kapton.

associated to the noncrystalline material (Figure 5, bottom). Then, according to plots of Figure 5, all the samples are semicrystalline including the thinnest sample (1:64) for which a weak 001 reflection can be seen (Figure 5, bottom).

The structural information like crystal orientation degree and crystal coherence size that can be derived from the shape of reflections ${ }^{22}$ is also relevant. In our case, the highly oriented GIWAXS patterns obtained deserve such analysis. Regarding reflection 001 it is considerably sharp in the azimuth direction. That would be related to crystal orientation. ${ }^{22}$ In Table 1, the full width at half-maximum (fwhm) values measured from the azimuth profiles of the 001 reflection are given. For samples as thin as $60 \mathrm{~nm}$, the fwhm values are similar and very small. That supports a high level of crystal orientation for these thinner samples as it comes out looking at the pattern of Figure 4. As mentioned above, our GIWAXS patterns exhibit an increasing background going from the top toward the equator. Due to such a background, azimuth profiles for 001 are deeply affected and they consist of a peak centered at $90^{\circ}$ which appears to be truncated on its base by symmetric tails of increasing intensity going away from the central peak (Figure 6). Therefore, we cannot properly determine the Herman orientation function ${ }^{23,24}$ in these oriented PPAz samples. Nevertheless, in the case of thinner samples

(22) Kakudo, M.; Kasay, N. X-ray diffraction by polymers; Kodansha Ltd.: Tokyo, 1972; Chapter 12.

(23) Balta-Calleja, F. J.; Vonk, C. G. X-ray scattering of synthetic polymers; Elsevier: Amsterdam, 1989; Chapter 6, p 215.

(24) Prasad, A.; Shroff, R.; Rane, S.; Beaucage, G. Morphological study of HDPE blown films by SAXS, SEM and TEM: a relationship between the melt elasticity parameter and lamellae orientation. Polymer 2001, 42(7), 3103-3113. 


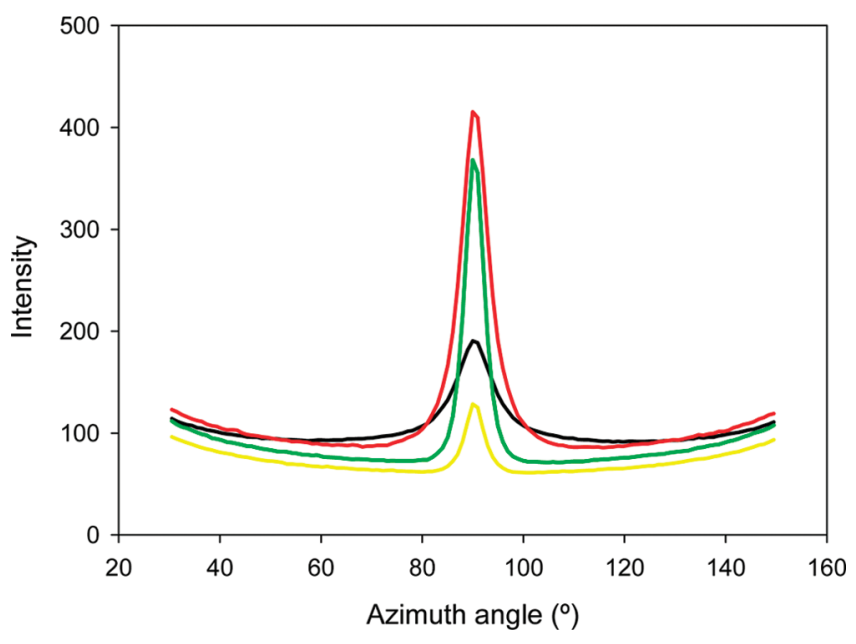

Figure 6. Azimuth intensity profiles for the 001 reflection of representative PPAz samples: (1:0) black, (1:2) red, (1:4) green, and (1:32) yellow. A cake width of 100 pixels was used to pick up the elongated 001 spot.

because the 001 reflection appears to be a spot without the arc (Figure 4), we can assume that the corresponding background matches the tails of their azimuth profiles (Figure 6). Then, a calculation of the Herman orientation function $f$ as described in ref 24 and taking as external reference axis the surface normal (meridian of GIWAXS pattern) was afforded. The derived $f$ values appear to be $\geq 0.99$ for all the samples of thickness $\leq 60 \mathrm{~nm}$. Therefore, these thinner samples exhibit a full lamellae orientation taking into account that $f=1$ means full or ideal crystal oriented sample while $f=0$ means isotropic or full disoriented sample. ${ }^{24}$

On the other side, the 001 reflection is relatively wide in the radial direction (see Figure 4 and plots of Figure 5). That is related to the crystal coherence length. ${ }^{22,25}$ Indeed, reflection 001 shows a fwhm value of about $1.1 \mathrm{~nm}^{-1}$ (in $q$ units), while reflection 010 is much sharper and shows a value of $0.32 \mathrm{~nm}^{-1}$. Taking into account the relationship ${ }^{25} \delta \beta_{h k l}=1 / D_{h k l}$, where $\delta \beta$ is the integral breadth of a $h k l$ reflection and $D$ is the crystal coherence length (crystal coherence size) along the normal direction to the $h \mathrm{kl}$ plane, and assuming that $\delta \beta \approx$ fwhm, the calculated crystal sizes are 5.7 and $19.6 \mathrm{~nm}$ along the [001] and [010] crystallographic directions, respectively $(q=2 \pi s$, with $s$ being the reciprocal vector). It has to be mentioned that the value $D_{001}=5.7 \mathrm{~nm}$ is similar to the thickness of the flat islands measured by AFM (Figure 2b). This means that the crystal lamella thickness is the coherent scattering length which comprises about 4 chain repeating units. Therefore, the reflection 001 ( $c^{*}$-axis) on the meridian of the GIWAXS patterns reveals that crystal lamellae are horizontal, in good agreement with the flat-on lamellae morphology observed by AFM.

3.3. Molecular Considerations about the Spin-Coating Morphology Developed in Linear Aliphatic Polyesters. As mentioned above, we have reported that the edge-on lamellae morphology is characteristic for spin-coating samples of aliphatic polyesters based on propylene glycol and a dicarboxylic acid of different length $\left(Y=2,3\right.$, and $\left.4 \mathrm{CH}_{2}\right) .{ }^{11}$ The results presented here for the homologous polymer PPAz with a longer dicarboxylic acid $\left(7 \mathrm{CH}_{2}\right)$, however, they clearly support a flat-on lamellae morphology, that is, lamellae parallel to the substrate. It is worth mentioning that we have used the same experimental conditions

(25) Balta-Calleja, F. J.; Vonk, C. G. X-ray scattering of synthetic polymers; Elsevier: Amsterdam, 1989; Chapter 4, p 141.

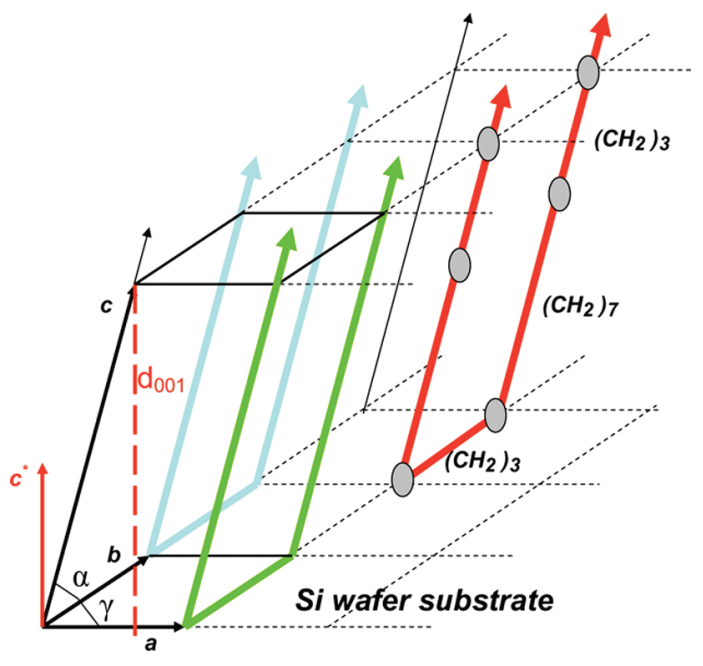

Figure 7. Schematic view of spin-coated PPAz chains on the Si substrate. Three polymer chains are marked with different colors. For the red one, the sequence of ester groups (ellipse symbol) linked by the short and long aliphatic spacers are indicated. The plane $a b$ of the crystal unit cell is parallel to the substrate, while the reciprocal axis $c^{*}$ is perpendicular to the substrate as result of the observed meridional reflection 001 whose lattice spacing $d_{001}$ is also shown. (Scheme is not at real length scale.)

in both studies about aliphatic polyesters prepared by spincoating: $\mathrm{CHCl}_{3}$ as solvent, polymer concentrations, Si-substrate (same wafers), and spin-coating procedures. According to the above results, we can say that PPAz becomes a good example to improve our understanding about the variables affecting both quality and morphology of spin-coating polymer thin films. PPAz chains consist of a sequence of ester groups (of moderate hydrophilic nature) separated by a short $\left(3 \mathrm{CH}_{2}\right)$ and a long $\left(7 \mathrm{CH}_{2}\right)$ aliphatic spacer of the propylene glycol and diacid moieties, respectively. Because of the hydrophilic nature of the substrate, it is plausible to assume a first anchoring of the chains to the substrate through the closer ester groups, that is, those linked by the propylene moiety. Due to the high length of the aliphatic spacer of the diacid and its repulsion by the hydrophilic substrate, it would provoke a redirection of the next long aliphatic spacers outward of the substrate where the closer ester groups are trapped. The stabilization of the chain segments going outward is certainly achieved by the subsequent crystallization of them. Taking into account the structural results of section 3.2 and the above comments, we think it will help the reader to see the schematic view of the PPAz chains on the substrate presented in Figure 7. On the one hand, the ability to crystallize of PPAz ${ }^{9}$ being much higher than that of the other aliphatic polyesters with shorter aliphatic sequences should also be responsible for the efficient coverage (continuous films) preventing the films from dewetting. On the other hand, the preferential edge-on lamellae morphology reported for the homologous aliphatic polyesters of shorter length ${ }^{11}$ should, therefore, be attributed to a more effective molecular interaction with the substrate of closer consecutive ester groups of the polymer chain. Consequently, polymer chains would prefer to lie parallel to the substrate in contrast to that observed here for PPAz.

\section{Conclusions}

Continuous thin films of PPAz (from 15 to $530 \mathrm{~nm}$ thick) have been prepared by spin-coating. The films exhibit a flat-on lamellae morphology which appears to be highly oriented for coating thicknesses below $60 \mathrm{~nm}$. The films are stable against dewetting even for thicknesses as thin as $15 \mathrm{~nm}$. Differences in both film 
stability and morphology of PPAz in relation to that reported for homologous aliphatic polyesters are attributed to a higher ability to crystallize of PPAz and its longer aliphatic spacer, respectively. The crystal coherence size, $D_{001}$ (about $5.7 \mathrm{~nm}$ ), seems to be coincident with the lamella thickness observed by AFM, particularly for the thinnest sample (1:64).
Acknowledgment. The authors are thankful for the financial support from the MICINN (Grant MAT2009-07789, MAT200803232, and FPU AP2005-1063), Spain. The experiments performed at BW4 in HASYLAB (DESY) were supported by the European Community (Contract RII3-CT-2004-506008(IA-SFS)). 